OPEN ACCESS

Edited by:

Kimberly Huey,

Drake University, United States

Reviewed by:

Ann L. Revill,

Midwestern University, United States

Jenna Monroy,

Claremont Colleges, United States

${ }^{*}$ Correspondence:

Tiffany J. Glass

glass@surgery.wisc.edu

Specialty section:

This article was submitted to

Exercise Physiology,

a section of the journal

Frontiers in Physiology

Received: 13 July 2021

Accepted: 27 September 2021

Published: 21 October 2021

Citation:

Glass TJ, Figueroa JE, Russell JA, Krekeler BN and Connor NP (2021)

Progressive Protrusive Tongue Exercise Does Not Alter Aging Effects

in Retrusive Tongue Muscles.

Front. Physiol. 12:740876.

doi: 10.3389/fphys.2021.740876

\section{Progressive Protrusive Tongue Exercise Does Not Alter Aging Effects in Retrusive Tongue Muscles}

\author{
Tiffany J. Glass ${ }^{1 *}$, Joanie E. Figueroa ${ }^{2}$, John A. Russell' ${ }^{1}$, Brittany N. Krekeler ${ }^{1,3}$ and \\ Nadine P. Connor ${ }^{1,4}$
}

1 Department of Surgery-Otolaryngology, University of Wisconsin-Madison, Madison, WI, United States, ${ }^{2}$ San Juan Bautista School of Medicine, Caguas, PR, United States, ${ }^{3}$ Department of Communication Sciences and Disorders, Northwestern University, Evanston, IL, United States, ${ }^{4}$ Department of Communication Sciences and Disorders, University of Wisconsin-Madison, Madison, WI, United States

Purpose: Exercise-based treatment approaches for dysphagia may improve swallow function in part by inducing adaptive changes to muscles involved in swallowing and deglutition. We have previously shown that both aging and progressive resistance tongue exercise, in a rat model, can induce biological changes in the genioglossus (GG); a muscle that elevates and protrudes the tongue. However, the impacts of progressive resistance tongue exercise on the retrusive muscles (styloglossus, SG; hyoglossus, HG) of the tongue are unknown. The purpose of this study was to examine the impact of a progressive resistance tongue exercise regimen on the retrusive tongue musculature in the context of aging. Given that aging alters retrusive tongue muscles to more slowly contracting fiber types, we hypothesized that these biological changes may be mitigated by tongue exercise.

Methods: Hyoglossus (HG) and styloglossus (SG) muscles of male Fischer 344/Brown Norway rats were assayed in age groups of young ( 9 months old, $n=24)$, middle-aged (24 months old, $n=23$ ), and old (32 months old, $n=26$ ), after receiving an 8-week period of either progressive resistance protrusive tongue exercise, or sham exercise conditions. Following exercise, HG and SG tongue muscle contractile properties were assessed in vivo. HG and SG muscles were then isolated and assayed to determine myosin heavy chain isoform (MyHC) composition.

Results: Both retrusive tongue muscle contractile properties and MyHC profiles of the $H G$ and $S G$ muscles were significantly impacted by age, but were not significantly impacted by tongue exercise. Old rats had significantly longer retrusive tongue contraction times and longer decay times than young rats. Additionally, HG and SG muscles showed significant MyHC profile changes with age, in that old groups had slower MyHC profiles as compared to young groups. However, the exercise condition did not induce significant effects in any of the biological outcome measures. 
Conclusion: In a rat model of protrusive tongue exercise, aging induced significant changes in retrusive tongue muscles, and these age-induced changes were unaffected by the tongue exercise regimen. Collectively, results are compatible with the interpretation that protrusive tongue exercise does not induce changes to retrusive tongue muscle function.

Keywords: tongue exercise, hyoglossus, styloglossus, rat, aging, muscle

\section{INTRODUCTION}

Normal swallowing function requires a series of precisely timed pressure changes within the upper aerodigestive tract that are accomplished primarily by tongue muscle contractile activity, which contributes to bolus containment and propulsion through the upper esophageal segment (Logemann, 1998; Lind, 2003). However, each year, 1 in 25 adults in the United States experience swallowing disorders (dysphagia) (Bhattacharyya, 2014). Prevalence increases with aging and age-related diseases with $50-75 \%$ of nursing home residents estimated to have dysphagia (Steele et al., 1997; O'Loughlin and Shanley, 1998), more than $80 \%$ of patients with Parkinson disease (Kalf et al., 2012; Suttrup and Warnecke, 2016), and $46-60 \%$ of patients with head and neck cancer (Shune et al., 2012). Swallowing impairment is common in elderly individuals (Eisenstadt, 2010), can significantly impact quality of life (Altman et al., 2010; Gassert and Pearson, 2016), and may lead to malnutrition (Altman et al., 2010), aspiration pneumonia (Martino et al., 2005) and dehydration (Smithard et al., 1996; Altman et al., 2010). Age-related changes in muscle composition have been shown to occur in tongue muscles (Rosenberg, 1997; Marzetti and Leeuwenburgh, 2006), with contractile and fiber properties shifting toward a slower contracting fiber profile with increasing age (Connor et al., 2008; Schaser et al., 2011). These changes may contribute to reduced tongue pressures and increased times to reach peak swallowing pressures in elderly persons, thereby increasing risks for dysphagia (Robbins et al., 1992, 1995; Nicosia et al., 2000). Exercise-based treatments to improve swallow function have been developed to target musculature involved in swallowing and deglutition. Since muscles of the tongue are highly active through bolus formation (Kayalioglu et al., 2007), bolus transport and propulsion (Van Daele et al., 2005; Palmer et al., 2008), tongue exercise has frequently been used clinically as a treatment for dysphagia (Robbins et al., 2005; Lazarus, 2006; Park et al., 2015; Rogus-Pulia et al., 2016; McKenna et al., 2017). This exercise involves having patients protrude and press their tongues against a fixed external structure, such as a tongue depressor or in more sophisticated approaches, against a pressure probe as is used in the Iowa Oral Performance Instrument (IOPI) ${ }^{\oplus}$ device (Robbins et al., 2005; Adams et al., 2013).

Tongue exercise paired with a water swallow has been modeled in the rat by our laboratory to study the effects of this intervention on tongue musculature (Connor et al., 2009; Kletzien et al., 2013; Krekeler and Connor, 2016; Schaser et al., 2016), with a degree of experimental precision that is not feasible in human subjects. Because of the predominant role of tongue protrusion in this exercise regimen, initial studies have focused on the genioglossus (GG) muscle, which is responsible for protrusion of the tongue (Bailey et al., 2007; Palmer et al., 2008). The hyoglossus (HG) and styloglossus (SG) are retrusive extrinsic tongue muscles. They receive their innervation from the lateral branch of the hypoglossal nerve (McClung and Goldberg, 2000), and contribute to lingual retraction (Car and Amri, 1987; Baer et al., 1988; Amri et al., 1989; Gilliam and Goldberg, 1995; Thexton et al., 1998; Napadow et al., 1999; Bailey and Fregosi, 2004). These muscles are active during a multitude of physiological functions such as contributions to airway patency, oral transport, swallowing, respiration, and speech (Hiiemae and Palmer, 2003; Kayalioglu et al., 2007; Liu et al., 2007). The relative contributions of retrusive and protrusive extrinsic musculature during protrusive lingual exercise have not been determined (Bailey et al., 2007; Palmer et al., 2008), but there is evidence that both protrusive (GG) and retrusive (SG, HG) tongue muscles are active during all phases of the oropharyngeal swallow (Amri et al., 1989; Napadow et al., 1999). Given that our laboratory's experimental protrusive exercise paradigm is paired with a water swallow, all extrinsic tongue muscles are likely activated during the course of the regimen to execute lingual press and swallow actions. In the rat model, a progressive resistance tongue exercise regimen has been shown to result in biological changes to the GG, including increased tongue muscle force generative capacity, trends in increasing muscle fiber size (Connor et al., 2009) and changes in myosin heavy chain composition (MyHC) (Kletzien et al., 2013). However, it is not known whether these measures of muscle biology are manifested in retrusive muscles of the tongue following protrusive tongue exercise.

Myosin heavy chain proteins (MyHC) are integral to the molecular contractile apparatus of muscle. There are a variety of myosin heavy chain protein isoforms, each with different functional properties linked to myofiber contractile velocity, contractile force, and ATPase activity. The contractile velocities and ATPase activities of skeletal muscle fibers correlate with the specific MyHC isoforms that they express, such that MyHC $2 \mathrm{~b}$ is associated with the fastest contraction velocities and greatest ATPase activity, followed by MyHC 2x, MyHC 2a, and finally, MyHC I, which is associated with comparatively slower contraction (Talmadge et al., 1993; Resnicow et al., 2010). Therefore, characteristics of skeletal muscle contraction speeds and fatigue depend in part upon MyHC isoform profiles which can be unique to each muscle type. MyHC isoform profiles may change in response to age and functional demands (Agbulut et al., 2003; Maejima et al., 2005; Yoshii et al., 2008), and therefore can provide a sensitive molecular indicator of how skeletal muscle 
properties change over time. While aging is generally associated with shifts in MyHC profiles, the nature of these shifts can vary with muscle type, such that some muscles reportedly adopt a faster $\mathrm{MyHC}$ profile with aging, while other muscles adopt a slower MyHC profile (Monemi et al., 1999). In the context of tongue muscles specifically, while aging results in slower MyHC profiles of the intrinsic tongue (Cullins and Connor, 2017), exercise may lead to shifts to faster $\mathrm{MyHC}$ profiles in some tongue regions (Cullins et al., 2018). Neuromuscular electrical stimulation (NMES) of the hypoglossal nerve has been found to alter HG and SG muscles, resulting in shifts toward slowercontracting $\mathrm{MyHC}$ isoform profiles, as well as longer contraction times and reduced muscle fatigue (Kletzien et al., 2018). However, NMES consists of highly controlled increases in tongue muscle activity, and therefore these prior findings may have limited applicability to the question of whether increases of tongue muscle activity through other means, such as voluntary tongue exercise, could similarly impact HG and SG muscles.

Thus, the purpose of this study was to examine the impacts of protrusive tongue exercise on the biology of the extrinsic, retrusive tongue musculature, specifically in the $\mathrm{HG}$ and SG muscles. We hypothesized that age would alter HG and SG muscles to slower muscle contraction times, with $\mathrm{MyHC}$ isoform profiles of more slowly contracting, fatigue-resistant forms, and that tongue exercise would mitigate these aging effects.

\section{MATERIALS AND METHODS}

\section{Protrusive Tongue Exercise}

This study was performed in accordance with the NIH Guide for Care and Use of Laboratory Animals (The National Academies, 2011), and the University of Wisconsin School of Medicine and Public Health Animal Care and Use Committee in Madison, Wisconsin (IACUC) approved the animal care and use protocol. In this study, 79 male Fischer 344 Brown Norway rats of 3 different ages [young adult ( 9 month), middle age (24 month) and old (32 month)] were divided into two groups: exercise and exercise sham (control). Exercise training was conducted on a reverse light cycle, to which rats were acclimated prior to training. Following this, rats were gradually acclimated over the course of a second week to conditions of water regulation, such that free access to water was provided for $3 \mathrm{~h}$ each day. Thereafter, rats were trained to use their tongue to press a disk in order to obtain a water reward. The baseline Estimated Maximum Press (EMP, average of the highest voluntary protrusive lingual pressing forces) of each rat was determined and was used to set individualized tongue press force thresholds for subsequent exercise. Rats were trained to elicit the water reward at progressively increased forces, as previously described (Kletzien et al., 2013; Krekeler and Connor, 2016). That is, rats were trained to press at $50 \%$ of EMP for two weeks, followed by $60 \%$ of EMP for the next two weeks. Midway through the second week of exercise at 60\% EMP, revised EMP values were determined for each rat based on midpoint EMP testing. This was followed by two weeks each of exercise at $70 \%$ of EMP and $80 \%$ of EMP, for a cumulative total of eight weeks of progressive tongue force exercise. During the eight weeks, rats were trained five days per week. For each exercise session, rats provided a minimum of 20 tongue presses per session at or above their respective force threshold during a time period of $10 \mathrm{~min}$. Following this, final EMP testing was completed over a three-day period to determine the final EMP force of each rat. Control rats received experimental conditions and handling that were similar to experimental rats, with the exception that during exercise sessions they were placed in the apparatus and allowed to lick or press briefly with trivial force in order to retain the behavioral task, then were promptly removed from the apparatus. That is, control rats were in the operandum until they did the task each session, but only to the minimum degree needed to keep them acclimated behaviorally to the task. However, control rats and experimental rats received the same procedures for EMP testing.

\section{Muscle Contractile Properties}

Retrusive tongue muscle contractile properties were analyzed as previously described (Becker et al., 2015; Kletzien et al., 2018). Briefly, rats were weighed, then deeply anesthetized with pentobarbitol administered intraperitoneally. Nerve cuff electrodes were placed bilaterally around the hypoglossal nerves. A silk suture was placed in the tip of the tongue, which was manually extended and connected to a force transducer. Optimal line tension on the suture was verified for each rat by adjusting the line tension to elicit the maximal twitch contraction. Hypoglossal nerves were stimulated bilaterally to generate retrusive tongue movements. Stimuli were delivered at $1 \mathrm{~Hz}$ to evaluate muscle twitch. Stimulation waveforms were.1-ms rectangular pulses at a supramaximally applied current, defined as 1.5 times the maximum current level required to obtain maximal twitch force (A-M Systems Differential AC amplifier, model 177; A-MSystems Isolated Pulse Stimulator, model 2100; A-M Systems Analog Stimulus Amplification Unit, model 2200, Carlsborg, WA). Data were electronically acquired directly into a dedicated computer using software developed for this purpose (Acquire 1.5; Madison, WI). Maximal twitch contraction time (the duration between the stimulus onset and 50\% peak twitch tension), twitch contraction half-decay time (the duration between the stimulus onset and $50 \%$ decay from peak twitch tension), and maximal twitch force (the peak tension elicited by a single stimulus) were analyzed. Tetanic forces were elicited through $200 \mathrm{~ms}$ trains with stimulus rates of 60,80 , and $100 \mathrm{~Hz}$, and maximal tetanic force (the maximal force of each stimulated fused wave) was recorded. After data collection, rats were euthanized and tissue was isolated for further analysis. Muscle samples were assigned alphanumeric codes to manage risks for unconscious experimental bias in subsequent molecular analyses.

\section{Sodium Dodecyl Sulfate-Polyacrylamide Gel Electrophoresis}

Muscle samples were stored in an $-80^{\circ} \mathrm{C}$ freezer until analysis. As previously described, muscle samples were homogenized and protein concentration was determined using a Bradford Protein Assay. $400 \mathrm{ng}$ protein was analyzed per sample through 
sodium dodecyl sulfate polyacrylamide gel electrophoresis (SDSPAGE) comprised of 0.75 -mm-thick $6 \%$ acrylamide $/ 30 \%$ glycerol separating gel $(18 \mathrm{~cm} \times 16 \mathrm{~cm})$ and a $4 \%$ acrylamide $/ 30 \%$ glycerol stacking gel, using the Hoefer SE 600 system (Amersham Biosciences, Piscataway, NJ). The running conditions were set at $275 \mathrm{~V}, 40 \mathrm{~mA}$, and $15 \mathrm{~W}$ for $24 \mathrm{~h}$ at $8^{\circ} \mathrm{C}$. The gels were stained and developed using Silver Quest Silver Staining Kit (Invitrogen, Carlsbad, CA) to visualize protein bands. As previously described, each $\mathrm{MyHC}$ isoform was identified through relative electrophoretic mobility $(2 \mathrm{a}<2 \mathrm{x}<2 \mathrm{~b}<\mathrm{I}$ ) (Kletzien et al., 2013). As a control for type I MyHC isoform, we used samples of soleus muscle, a predominately slow twitch muscle in the rat, and for type MyHC2a, MyHC2x, MyHC2b we used samples of extensor digitorum longus (EDL) muscle, a predominately fast twitch muscle in rat.

\section{Statistical Analysis}

One-way Analysis of Variance (ANOVA) was performed to evaluate the impact of age on baseline EMP values at initial tongue press testing. Two-way ANOVAs were performed to determine the impact of independent variables (exercise and age) on the dependent biological measures of estimated maximum press force, myosin heavy chain isoform profiles, decay time, and twitch time. ANOVAs were performed using Graphpad Prism v9 (La Jolla, CA). The measures of maximum tetanic force and maximum twitch force were analyzed using IBM SPSS Statistics v.25, through ANCOVA with body weight as a covariate, in order to accommodate the possibility that body weight influences muscle contraction force measurements (Hurd et al., 2011). The mean body weights (SD) of groups on the day of euthanasia were: Young Adult Control: 406.1 (16.9) g, Young Adult Exercise: 400.5 (20.11) g, Middle Control: 500.3 (43.2) g, Middle Exercise: 498.8 (30.2) g, Old Control: 450.5 (37.49) g, Old Exercise: 448.1 (53.73) g. No data points were removed from analysis, although incidental loss due to technical artifact, health-related attrition or sample loss resulted in unequal group sizes in some experiments. Therefore, of the 79 rats originally assigned to this study, 73 rats ultimately contributed data for this study. Significance was defined at $\alpha=0.05$. Statistical tests are summarized in Supplementary Table 1.

\section{RESULTS}

\section{Protrusive Tongue Exercise}

At baseline, EMP were not significantly different between the age groups of old $($ Mean $=50.35 \mathrm{mN}, \mathrm{SD} 15.81)$, middle $($ Mean $=56.79 \mathrm{mN} \mathrm{SD} \mathrm{19.16)}$ and young $($ Mean $=53.86 \mathrm{mN}$ SD 14.59). Final EMP forces at the completion of the protrusive tongue exercise regimen were significantly greater in the exercise condition (Mean of all ages $=124.8 \mathrm{mN}$, SD 36.4) than the control condition (Mean of all ages $=62.88 \mathrm{mN}, \mathrm{SD} 16.35$ ). Following the protrusive tongue exercise regimen, mean tongue press force increases (delta EMP) of exercise groups were collectively 5.8fold greater than mean tongue press force increases of control groups which received sham exercise. Separated by age, final EMP forces were $60.78 \mathrm{mN}$ greater than baseline in the young

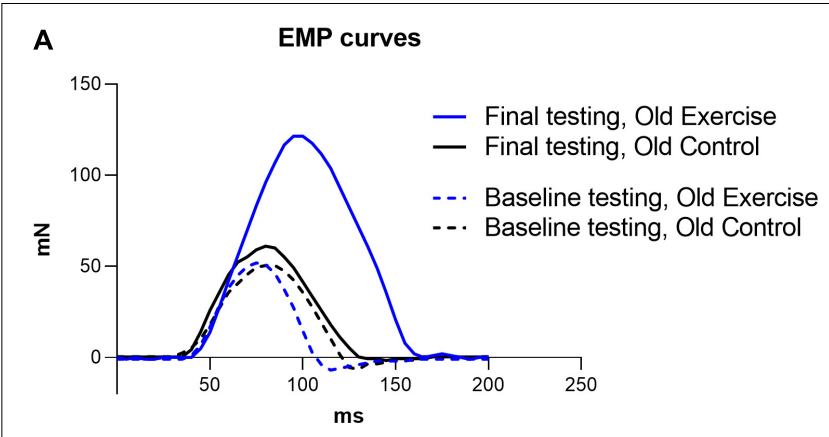

B Changes in Tongue Force after Exercise

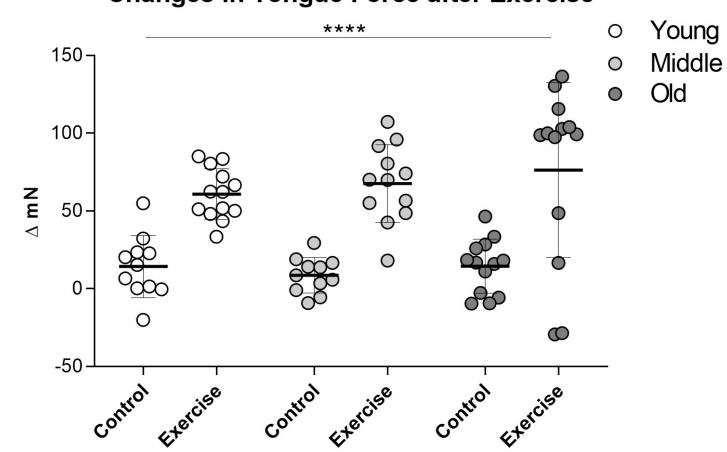

FIGURE 1 | Change in EMP (Estimated Maximum Press) before and after exercise regimen. (A) Examples of tongue press force curves before and after tongue exercise in one old control rat and one old exercise rat. (B) Quantification of changes in Estimated Maximum Press (EMP) forces between initial and final tongue press testing. Results were significant for exercise condition, but not for age. Each symbol indicates data for one rat. Plots indicate group means and SD. Group sizes: Young Adult Control $=11$, Young Adult Exercise $=13$, Middle Aged Control $=11$, Middle Exercise $=12$, Old Aged Control $=13$, Old Aged Exercise $=13 .{ }^{* \star * \star}=P<0.0001$.

adult exercise group, $67.52 \mathrm{mN}$ greater than baseline in middle aged adult exercise group, and $76.26 \mathrm{mN}$ greater than baseline in the old exercise group. Analyses of changes in tongue force (delta EMP) between the beginning and the end of the 8-week exercise regimen confirmed a highly significant exercise effect $(p<0.0001)$, in the absence of a significant age effect, and in the absence of significant interactions between age and exercise (Figures 1A,B).

\section{Retrusive Muscle Contractile Properties}

There was a significant main effect for age on some measures of retrusive muscle contractile properties. There was not a significant interaction effect between age and exercise, and the exercise condition did not have a significant effect on retrusive muscle contractile properties. Irrespective of the exercise condition, maximum twitch force was significantly greater in the old (Mean $=310.4 \mathrm{mN}, \mathrm{SD} 44.41)$ than middle aged (Mean $=293.5 \mathrm{mN}$, SD 32.55) or young adult groups $($ Mean $=277.9 \mathrm{mN}, \mathrm{SD} 24.94)(p=0.01)$ (Figures 2A,B). However, age had no significant effect on the Maximum Tetanic Force $(p=0.116)$ (Figure 2C). There was a significant main effect for age on half-decay time, such that the old group had 
longer mean half-decay times (Mean $=36.73 \mathrm{~ms}$, SD 3.62) than middle aged (Mean $=34.89 \mathrm{~ms}$, SD 1.61) or young groups $($ Mean $=34.44 \mathrm{~ms}$, SD 1.413 $)(p=0.004)$ (Figure 2D). There was also a significant main effect for age on muscle contraction time, with the old group having longer mean contraction times $($ Mean $=10.05 \mathrm{~ms}, \mathrm{SD} .82)$ than middle aged $($ Mean $=9.25 \mathrm{~ms}$, $\mathrm{SD} .44$ ) or young adult groups (Mean $=9.14 \mathrm{~ms}$, SD .43), $(p<0.0001)$ (Figure 2E).

\section{Myosin Heavy Chain Composition Profiles}

Analysis of MyHC profiles for $\mathrm{HG}$ and SG muscles revealed significant main effects for age, and no significant effects for exercise, in the absence of interactions between age and exercise. In the $\mathrm{HG}$ muscle, MyHC isoforms showing significant differences across age were MyHC 2x $(p=0.013)$, and $\mathrm{MyHC}$ $2 \mathrm{~b}(p=0.004)$ (Figures 3A-D). The young adult group showed proportionally greater amounts of $\mathrm{MyHC} 2 \mathrm{~b}$ (Mean $=43.13 \%$, SD $8.85)$ than middle aged (Mean $=37.42 \%$, SD 2.911 ) or old groups (Mean $=38.01 \%$, SD 2.50). In the HG, the young adult group also showed less MyHC 2x (Mean $=37.61 \%$, SD 4.65) than middle aged $($ Mean $=41.16 \%$, SD 4.09$)$ or old groups $($ Mean $=41.34 \%$, $\mathrm{SD}$ 8.85). In the $\mathrm{SG}$ muscle, there were significant increases in the relative proportion of $\mathrm{MyHC} 2 \mathrm{a}$ with age $(p=0.001)$. The SG in the young adult group showed proportionately lower MyHC 2a content (Mean $=23.49 \%$, SD 4.52) than middle aged $($ Mean $=28.75 \%$, SD 4.95$)$ or old groups (Mean $=27.95 \%$, $\mathrm{SD}$ 3.96). There were also significant reductions in relative proportions of MyHC $2 \mathrm{~b}$ with age, such that the mean MyHC $2 \mathrm{~b}$ content of SG muscles was greater in the young adult group $($ Mean $=34.05 \%$, SD 4.37) than middle aged $($ Mean $=26.87 \%$, SD $7.39)$ or old adult groups $($ Mean $=26.65 \%$, SD 5.12) $(p=0.0002)$ (Figures 3E-H). While small levels of $\mathrm{MyHC}$ I were present in SG and HG muscles of some rats, relative levels of this isoform were generally scant or undetectable (Figures 3A,E), and were not significantly different between groups.

\section{DISCUSSION}

This work tested the hypothesis that aging would impact biological properties of retrusive tongue muscles in a rat model, and that a protrusive tongue exercise regimen would ameliorate these aging effects. Rats of three different age groups underwent eight weeks of a progressive tongue exercise regimen, after which behavioral tongue forces significantly increased. At the completion of this exercise regimen, retrusive tongue muscle contractile properties were evaluated in vivo through whole hypoglossal nerve stimulation, after which the HG and SG muscles were isolated and evaluated for MyHC protein profiles. It was found that although age significantly impacted retrusive tongue contractile properties and $\mathrm{MyHC}$ to more slowly contracting profiles, protrusive progressive resistance tongue exercise did not significantly impact either retrusive tongue contractile properties nor retrusive muscle $\mathrm{MyHC}$ profiles.

The finding of significant effects of age on retrusive tongue muscle contractile properties and $\mathrm{MyHC}$ profiles in the present study is not unexpected in light of several prior reports describing age-related changes in tongue muscles (Kletzien et al., 2013; Cullins and Connor, 2017). As in the present study, a prior study identified significantly longer retrusive contraction times upon hypoglossal nerve stimulation in old rats than for young adult rats (Becker et al., 2015). These age-related differences in muscle contractile function are broadly mirrored by age-related differences in muscle MyHC profiles. Prior studies have reported that with age, the GG muscle shows MyHC shifts indicative of more slowly contracting, fatigue-resistant fiber types, such that the GG of old rats may have relatively greater proportions of $\mathrm{MyHC}$, and relatively lower proportions of $\mathrm{MyHC} 2 \mathrm{~b}$ than young rats (Connor et al., 2013). Similarly, both the HG and SG muscles in the present study demonstrated shifts toward more slowly contracting, fatigue-resistant fiber types with age, such that the retrusive tongue muscles contained lower relative proportions of $\mathrm{MyHC} 2 \mathrm{~b}$ in old rats as compared to young rats. These findings are broadly corroborated by earlier studies which also reported significant $\mathrm{MyHC}$ changes in the retrusive extrinsic tongue muscles with age (Connor et al., 2013; Kletzien et al., 2018).

The absence of a detectible effect of tongue exercise on retrusive tongue muscle measures is compatible with the interpretation that the progressive resistance tongue exercise regimen may have a high degree of specificity in direct muscular impact. Tongue protrusion is largely accomplished through contraction of the genioglossus muscle, while synergistic contraction of the transverse and verticalis muscles of the intrinsic tongue are believed to result in tongue elongation (McClung and Goldberg, 2000). Tongue retrusion is believed to be accomplished through contraction of the hyoglossus, styloglossus, and the superior and inferior longitudinal intrinsic tongue muscles (McClung and Goldberg, 2000). However, it is also known that co-activation of both muscles of tongue protrusion and muscles of tongue retrusion may be important for a variety of movements and functions (Fregosi and Fuller, 1997; Fuller et al., 1999). While most muscles of the body have bony or cartilaginous origins and insertions, the tongue muscle system is unique in that it is generally understood to function as a muscular hydrostat, such that it likely depends on coactivation of muscles for support and stability in movement to a degree that is not seen in limb muscles (Gilbert et al., 2007). Both intrinsic and extrinsic muscles of the tongue interdigitate and work synergistically to accomplish tongue protrusion, tongue retrusion, and stabilization (Fuller et al., 1999; McClung and Goldberg, 2000; Gilbert et al., 2007), and therefore it is conceivable that some tongue movements may involve concurrent activation of both protrusive and retrusive tongue muscles (Gilbert et al., 2007). Analysis of exerciseinduced $\mathrm{MyHC}$ changes in discrete tongue muscles is one way through which evidence may be obtained corroborating either the involvement or exclusion of different tongue muscles in specific tongue movements (Cullins et al., 2018). While investigations of impact of lingual exercise on protrusive tongue musculature (genioglossus) have demonstrated mixed findings (Kletzien et al., 2013; Cullins et al., 2018; Krekeler et al., 2020), current findings of unaltered biological measures of 

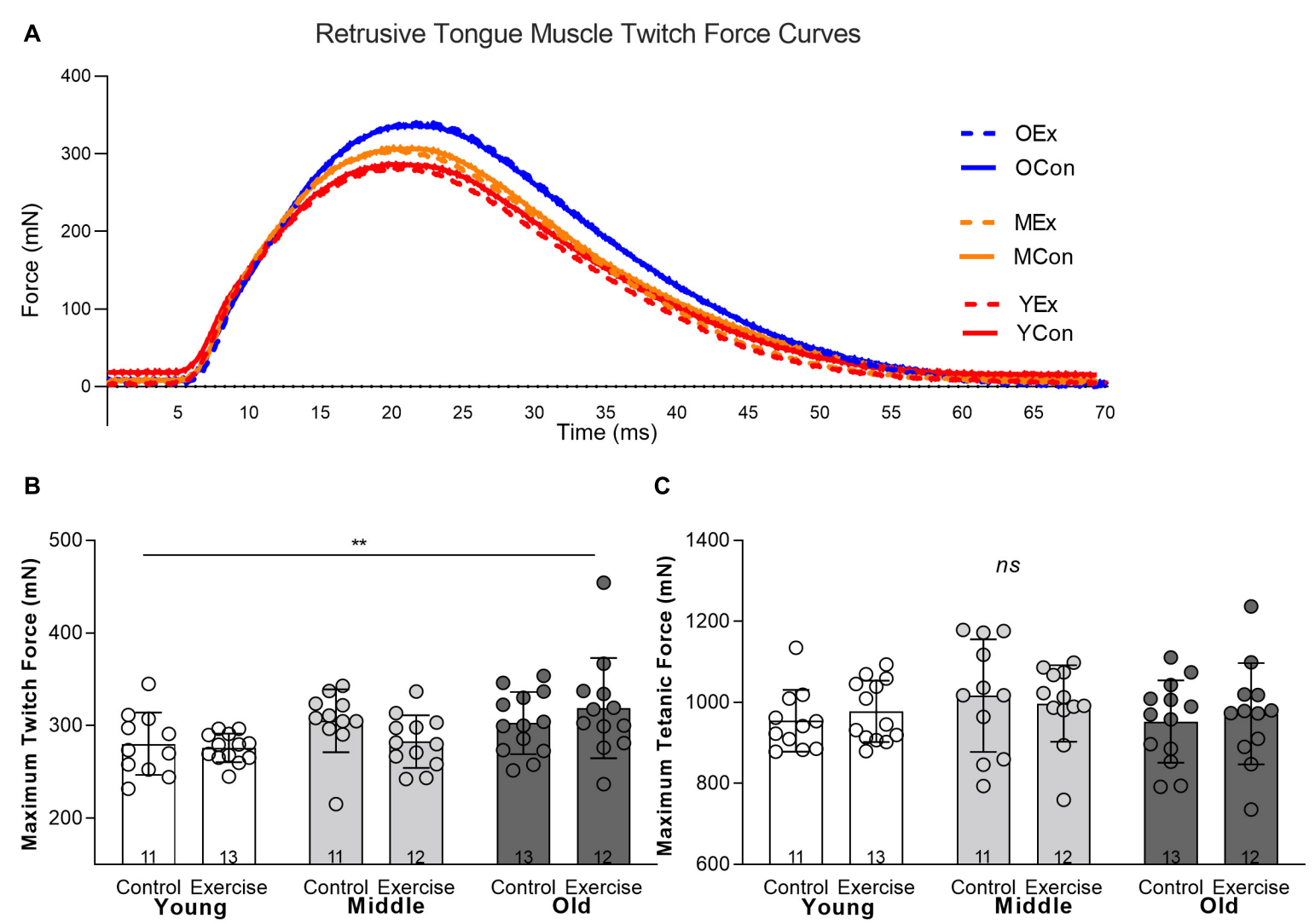

D

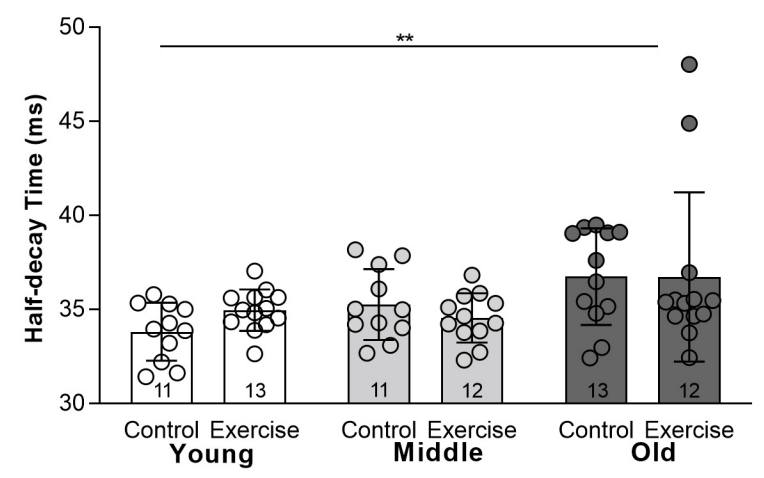

E

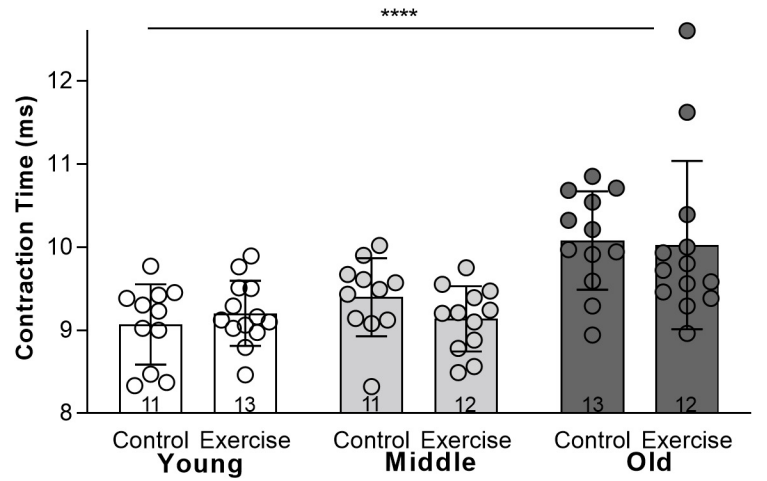

FIGURE 2 | In vivo retrusive tongue muscle contractile properties showed significant differences for age, but were not significantly impacted by exercise. (A) Example twitch force curves are shown for one individual rat from each of the six experimental groups. YEx = Young adult, Exercise, YCon $=$ Young adult, Control, MEx = Middle aged, Exercise, MCon = Middle aged, Control, OEx = Old age, Exercise, OCon = Old age, Control. (B) Maximum Twitch Force (C) Maximum Tetanic Force, (D) Half-decay Time, (E) Contraction Time. Bars show mean and SD, with specific group sample sizes indicated. Each symbol indicates data for one rat. ${ }^{\star * * \star}=P<0.0001,{ }^{* *}=P<0.01, \mathrm{~ns}=$ no significant differences.

retrusive tongue muscles following tongue exercise suggest that progressive resistance protrusive tongue exercise does not significantly impact the extrinsic muscles of tongue retrusion. These findings suggest that inducing biological change via oromotor exercise may require highly specific movements, or practice geared toward activating muscular groups required for the desired action.
There are limitations to this study. While rat models of oromotor functions are invaluable for translational research (German et al., 2017), it should be noted that some intrinsic differences in tongue function between rats and humans may impose certain limitations on the translational applicability of this study. Depending on the species and the context, the ways mammals use the tongue on a daily basis can vary in 


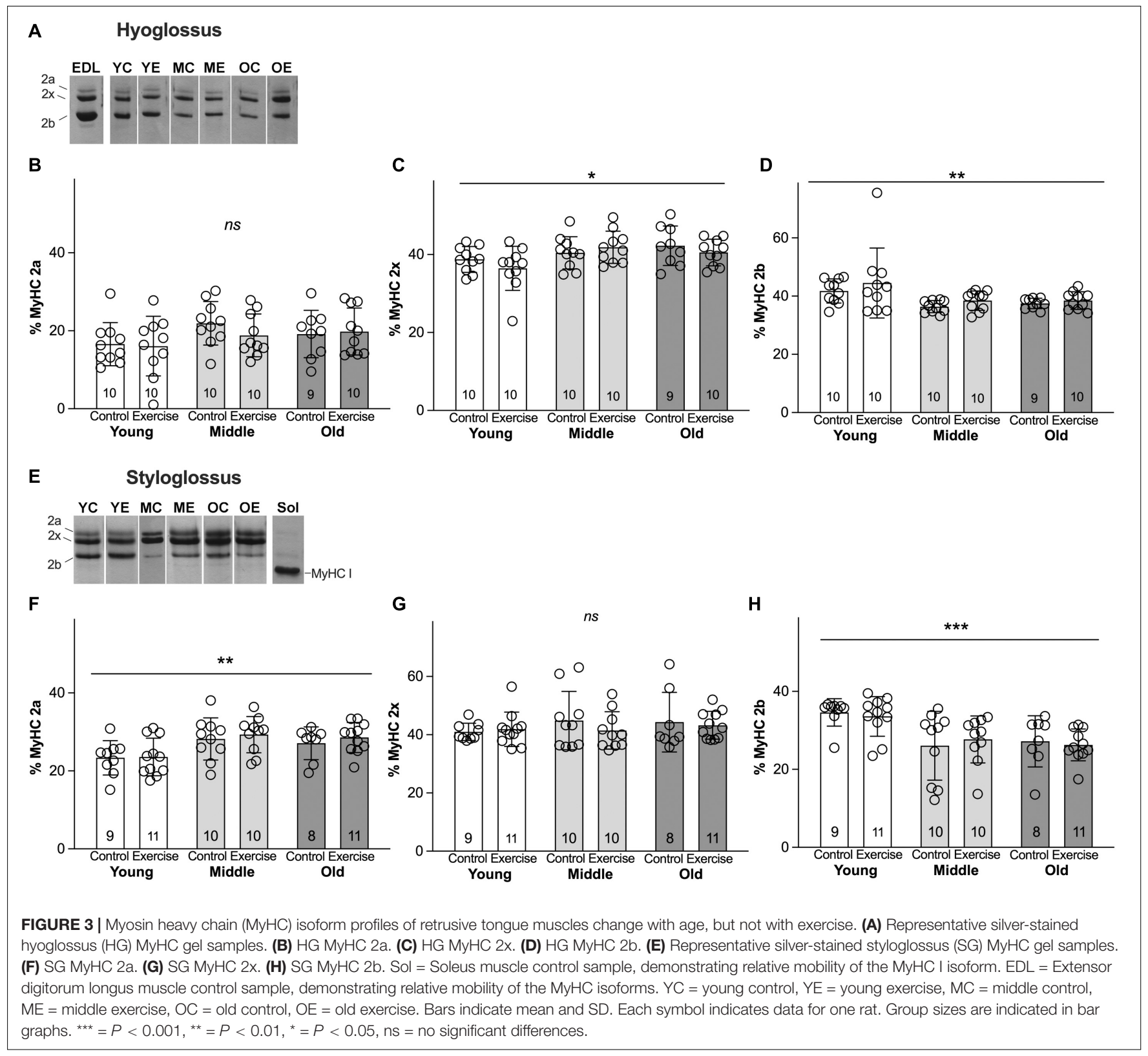

the magnitude of protrusive tongue movements and retrusive tongue movements (Olson et al., 2021). Notably, while humans typically drink with their tongue positioned intraorally (Matsuo and Palmer, 2008), rats typically drink by protruding the tongue from the oral cavity while licking or lapping (Weijnen, 1998). This constitutes a difference in the utility and magnitude of tongue protrusion and retrusion in rats as compared to humans. Further, while the tongue exercise paradigm studied here involves protrusion of the tongue from the oral cavity to exert progressive force on an external object, tongue exercise regimen in humans are often likely to involve exercises with intraoral tongue movements (Yoshikawa et al., 2021). Therefore, it is possible that rats and humans may be anticipated to differ somewhat in the degree to which retrusive tongue muscles are impacted by a given tongue exercise regimen. These limitations notwithstanding, the biological investigations of discrete tongue muscles that are possible in rat models can provide anatomically precise information that cannot be obtained from humans.

This current study's findings that protrusive tongue exercise does not impact aging effects in retrusive tongue muscles suggests two implications. First, there is interest in the possibility that tongue exercise regimen can impact or improve swallow function (Kim et al., 2017; Krekeler et al., 2020), although mechanisms through which that may occur remain somewhat unclear. It is known that protrusive tongue muscles play important roles in the oral stage of swallowing, and therefore increased strength or altered function of protrusive muscles may be a means through which tongue 
exercise could produce improved outcomes (Kim et al., 2017), conceivably in the absence of increased strength or altered function of retrusive tongue muscles. Secondly, these results also inspire the question of whether future experimental work in therapeutic tongue exercise regimen could be modified to target retrusive muscles. It is known that retrusive tongue muscles are integral to retrograde bolus propulsion during swallowing (Gilbert et al., 2007). Although the protrusive tongue exercise task in the current study coincided with a water swallow, modulation of the retrusive movements involved in swallowing was not a direct focus of the regimen. Future investigations using rat paradigms should examine requirements for inducing biological change in targeted muscular groups that will contribute to optimize functional improvements in deglutition and swallowing, including mechanisms of adaptive responses to exercise dose, and fatigue prevention.

\section{DATA AVAILABILITY STATEMENT}

The original contributions presented in the study are included in the article/Supplementary Material, further inquiries can be directed to the corresponding author.

\section{ETHICS STATEMENT}

The animal study was reviewed and approved by University of Wisconsin School of Medicine and Public Health Animal Care and Use Committee in Madison, Wisconsin (IACUC).

\section{REFERENCES}

Adams, V., Mathisen, B., Baines, S., Lazarus, C., and Callister, R. (2013). A systematic review and meta-analysis of measurements of tongue and hand strength and endurance using the Iowa Oral Performance Instrument (IOPI). Dysphagia 28:350. doi: 10.1007/s00455-013-9451-3

Agbulut, O., Noirez, P., Beaumont, F., and Butler-Browne, G. (2003). Myosin heavy chain isoforms in postnatal muscle development of mice. Biol. Cell 95, 399-406. doi: 10.1016/S0248-4900(03)00087-X

Altman, K. W., Yu, G.-P., and Schaefer, S. D. (2010). Consequence of dysphagia in the hospitalized patient: impact on prognosis and hospital resources. Arch. Otolaryngol.Head Neck Surg. 136, 784-789. doi: 10.1001/archoto.2010.129

Amri, M., Lamkaden, M., and Car, A. (1989). Activity of extrinsic tongue muscle during swallowing in sheep. Brain Res. 503, 141-143. doi: 10.1016/00068993(89)91714-9

Baer, T., Alfonso, P. J., and Honda, K. (1988). Ann. bull. rilp (1988) no. 22, 7-19 electromyography of the tongue muscles during vowels in/gpvp/environment. Ann. Bull. RILP 22, 7-19.

Bailey, E. F., and Fregosi, R. F. (2004). Coordination of intrinsic and extrinsic tongue muscles during spontaneous breathing in the rat. J. Appl. Physiol. 96, 440-449. doi: 10.1152/japplphysiol.00733.2003

Bailey, E. F., Rice, A. D., and Fuglevand, A. J. (2007). Firing patterns of human genioglossus motor units during voluntary tongue movement. J. Neurophysiol. 97, 933-936. doi: 10.1152/jn.00737.2006

Becker, B. J., Russell, J. A., and Connor, N. P. (2015). Effects of aging on evoked retrusive tongue actions. Arch. Oral Biol. 60, 966-971. doi: 10.1016/j. archoralbio.2015.02.025

Bhattacharyya, N. (2014). The prevalence of dysphagia among adults in the United States. Otolaryngol. Head Neck Surg. 151, 765-769. doi: 10.1177/ 0194599814549156

\section{AUTHOR CONTRIBUTIONS}

NC conceived of the study and provided the requisite resources. JF, TG, and JR performed experiments in the study. TG, JF, and JR compiled and analyzed the data. JF, TG, JR, BK, and NC wrote and edited the manuscript. All authors contributed to the article and approved the submitted version.

\section{FUNDING}

The authors gratefully acknowledge the following funding sources which made this work possible: NIH, National Institute on Deafness and Other Communication Disorders (NIDCD) R01 DC018071 (NC), DC008149 (NC), T32 DC009401 (JF), and NIH, National Cancer Institute (NCI) R37 CA225608 (JR).

\section{ACKNOWLEDGMENTS}

We wish to acknowledge the important contributions of Jared Cullen in oversight of rat training and assistance with data compilation.

\section{SUPPLEMENTARY MATERIAL}

The Supplementary Material for this article can be found online at: https://www.frontiersin.org/articles/10.3389/fphys. 2021.740876/full\#supplementary-material

Car, A., and Amri, M. (1987). Activity of neurons located in the region of the hypoglossal motor nucleus during swallowing in sheep. Exp. Brain Res. 69, 175-182. doi: 10.1007/BF00247040

Connor, N. P., Ota, F., Nagai, H., Russell, J. A., and Leverson, G. (2008). Differences in age-related alterations in muscle contraction properties in rat tongue and hindlimb. J. Speech Lang. Hearing Res. 51, 818-827. doi: 10.1044/ 1092-4388(2008/059)

Connor, N. P., Russell, J. A., Jackson, M. A., Kletzien, H., Wang, H., Schaser, A. J., et al. (2013). Tongue muscle plasticity following hypoglossal nerve stimulation in aged rats. Muscle Nerve 47, 230-240. doi: 10.1002/mus.23499

Connor, N. P., Russell, J. A., Wang, H., Jackson, M. A., Mann, L., and Kluender, K. (2009). Effect of tongue exercise on protrusive force and muscle fiber area in aging rats. J. Speech Lang. Hearing Res. JSLHR 52, 732-744. doi: 10.1044/10924388(2008/08-0105)

Cullins, M. J., and Connor, N. P. (2017). Alterations of intrinsic tongue muscle properties with aging. Muscle Nerve 2017:25605. doi: 10.1002/mus.25605

Cullins, M. J., Krekeler, B. N., and Connor, N. P. (2018). Differential impact of tongue exercise on intrinsic lingual muscles. Laryngoscope 128, 2245-2251. doi: 10.1002/lary.27044

Eisenstadt, S. (2010). Dysphagia and aspiration pneumonia in older adults. J. Am. Acad. Nurse Practit. 22, 17-22. doi: 10.1111/j.1745-7599.2009.00470.x

Fregosi, R. F., and Fuller, D. D. (1997). Respiratory-related control of extrinsic tongue muscle activity. Respir. Physiol. 110, 295-306. doi: 10.1016/S00345687(97)00095-9

Fuller, D. D., Williams, J. S., Janssen, P. L., and Fregosi, R. F. (1999). Effect of coactivation of tongue protrudor and retractor muscles on tongue movements and pharyngeal airflow mechanics in the rat. J. Physiol. 519(Pt 2), 601-613. doi: 10.1111/j.1469-7793.1999.0601m.x

Gassert, R. B., and Pearson, W. G. Jr. (2016). Evaluating muscles underlying tongue base retraction in deglutition using muscular functional magnetic resonance 
imaging (mfMRI). Magnet. Resonance Imaging. 34, 204-208. doi: 10.1016/j.mri. 2015.10.029

German, R. Z., Crompton, A. W., Gould, F. D., and Thexton, A. J. (2017). Animal Models for Dysphagia Studies: What Have We Learnt So Far. Dysphagia 32, 73-77. doi: 10.1007/s00455-016-9778-7

Gilbert, R. J., Napadow, V. J., Gaige, T. A., and Wedeen, V. J. (2007). Anatomical basis of lingual hydrostatic deformation. J. Exp. Biol. 210(Pt 23), 4069-4082. doi: $10.1242 /$ jeb.007096

Gilliam, E. E., and Goldberg, S. J. (1995). Contractile properties of the tongue muscles: effects of hypoglossal nerve and extracellular motoneuron stimulation in rat. J. Neurophysiol. 74, 547-555. doi: 10.1152/jn.1995.74.2.547

Hiiemae, K. M., and Palmer, J. B. (2003). Tongue movements in feeding and speech. Crit. Rev. Oral Biol. Med. 14, 413-429. doi: 10.1177/154411130301400604

Hurd, W. J., Morrey, B. F., and Kaufman, K. R. (2011). The effects of anthropometric scaling parameters on normalized muscle strength in uninjured baseball pitchers. J. Sport Rehabil. 20, 311-320. doi: 10.1123/jsr.20.3.311

Kalf, J., De Swart, B., Bloem, B., and Munneke, M. (2012). Prevalence of oropharyngeal dysphagia in Parkinson's disease: a meta-analysis. Parkinsonism Related Disord. 18, 311-315. doi: 10.1016/j.parkreldis.2011.11.006

Kayalioglu, M., Shcherbatyy, V., Seifi, A., and Liu, Z. J. (2007). Roles of intrinsic and extrinsic tongue muscles in feeding: electromyographic study in pigs. Arch. Oral Biol. 52, 786-796. doi: 10.1016/j.archoralbio.2007.01.004

Kim, H. D., Choi, J. B., Yoo, S. J., Chang, M. Y., Lee, S. W., and Park, J. S. (2017). Tongue-to-palate resistance training improves tongue strength and oropharyngeal swallowing function in subacute stroke survivors with dysphagia. J. Oral Rehabil. 44, 59-64. doi: 10.1111/joor.12461

Kletzien, H., Russell, J. A., Leverson, G. E., and Connor, N. P. (2013). Differential effects of targeted tongue exercise and treadmill running on aging tongue muscle structure and contractile properties. J. Appl. Physiol. 114, 472-481. doi: 10.1152/japplphysiol.01370.2012

Kletzien, H., Russell, J. A., Leverson, G., and Connor, N. P. (2018). Effect of neuromuscular electrical stimulation frequency on muscles of the tongue. Muscle Nerve 58, 441-448. doi: 10.1002/mus.26173

Krekeler, B. N., and Connor, N. P. (2016). Age-related changes in mastication are not improved by tongue exercise in a rat model. Laryngoscope 127, E29-E34. doi: $10.1002 /$ lary. 26045

Krekeler, B. N., Weycker, J. M., and Connor, N. P. (2020). Effects of Tongue Exercise Frequency on Tongue Muscle Biology and Swallowing Physiology in a Rat Model. Dysphagia 12, 918-934. doi: 10.1007/s00455-020-10105-2

Lazarus, C. (2006). Tongue strength and exercise in healthy individuals and in head and neck cancer patients. New York, NY: Thieme Medical Publishers, Inc., 260-267. doi: 10.1055/s-2006-955116

Lind, C. D. (2003). Dysphagia: evaluation and treatment. Gastroenterol. Clin. North Am. 32, 553-575. doi: 10.1016/S0889-8553(03)00024-4

Liu, Z. J., Kayalioglu, M., Shcherbatyy, V., and Seifi, A. (2007). Tongue deformation, jaw movement and muscle activity during mastication in pigs. Arch. Oral Biol. 52, 309-312. doi: 10.1016/j.archoralbio.2006.10.024

Logemann, J. A. (1998). The evaluation and treatment of swallowing disorders. Curr. Opin. Otolaryngol. Head Neck Surg. 6, 395-400. doi: 10.1097/00020840199812000-00008

Maejima, M., Abe, S., Sakiyama, K., Agematsu, H., Hashimoto, M., Tamatsu, Y., et al. (2005). Changes in the properties of mouse tongue muscle fibres before and after weaning. Arch. Oral Biol. 50, 988-993. doi: 10.1016/j.archoralbio.2005 03.007

Martino, R., Foley, N., Bhogal, S., Diamant, N., Speechley, M., and Teasell, R. (2005). Dysphagia after stroke: incidence, diagnosis, and pulmonary complications. Stroke J. Cereb. Circulat. 36, 2756-2763. doi: 10.1161/01.STR. 0000190056.76543.eb

Marzetti, E., and Leeuwenburgh, C. (2006). Skeletal muscle apoptosis, sarcopenia and frailty at old age. Exp. Gerontol. 41, 1234-1238. doi: 10.1016/j.exger.2006. 08.011

Matsuo, K., and Palmer, J. B. (2008). Anatomy and physiology of feeding and swallowing: normal and abnormal. Phys. Med. Rehabil. Clin. N. Am. 19, 691707. doi: $10.1016 /$ j.pmr.2008.06.001

McClung, J. R., and Goldberg, S. J. (2000). Functional anatomy of the hypoglossal innervated muscles of the rat tongue: a model for elongation and protrusion of the mammalian tongue. Anat. Rec. 260, 378-386. doi: 10.1002/10970185(20001201)260:4<378::AID-AR70>3.0.CO;2-A
McKenna, V. S., Zhang, B., Haines, M. B., and Kelchner, L. N. (2017). A Systematic Review of Isometric Lingual Strength-Training Programs in Adults With and Without Dysphagia. Am. J. Speech Lang. Pathol. 26, 524-539. doi: 10.1044/ 2016_AJSLP-15-0051

Monemi, M., Eriksson, P. O., Kadi, F., Butler-Browne, G. S., and Thornell, L. E. (1999). Opposite changes in myosin heavy chain composition of human masseter and biceps brachii muscles during aging. J. Muscle Res. Cell Motil. 20, 351-361. doi: 10.1023/A:1005421604314

Napadow, V. J., Chen, Q., Wedeen, V. J., and Gilbert, R. J. (1999). Biomechanical basis for lingual muscular deformation during swallowing. Am. J. Physiol. Gastrointest. Liver Physiol. 277, G695-G701. doi: 10.1152/ajpgi.1999.277.3. G695

Nicosia, M. A., Hind, J. A., Roecker, E. B., Carnes, M., Doyle, J., Dengel, G. A., et al. (2000). Age effects on the temporal evolution of isometric and swallowing pressure. J. Gerontol. Biol. Sci. Med. Sci. 55, M634-M640. doi: 10.1093/gerona/ 55.11.M634

O'Loughlin, G., and Shanley, C. (1998). Swallowing problems in the nursing home: a novel training response. Dysphagia 13, 172-183. doi: 10.1007/PL00009569

Olson, R. A., Montuelle, S. J., Chadwell, B. A., Curtis, H., and Williams, S. H. (2021). Jaw kinematics and tongue protraction-retraction during chewing and drinking in the pig. J. Exp. Biol. 224:239509. doi: 10.1242/jeb.239509

Palmer, P. M., Jaffe, D. M., McCulloch, T. M., Finnegan, E. M., Van Daele, D. J., and Luschei, E. S. (2008). Quantitative contributions of the muscles of the tongue, floor-of-mouth, jaw, and velum to tongue-to-palate pressure generation. J. Speech Lang. Hearing Res. 51, 828-835. doi: 10.1044/1092$4388(2008 / 060)$

Park, J.-S., Kim, H.-J., and Oh, D.-H. (2015). Effect of tongue strength training using the Iowa Oral Performance Instrument in stroke patients with dysphagia. J. Phys. Therapy Sci. 27, 3631-3634. doi: 10.1589/jpts.27.3631

Resnicow, D. I., Deacon, J. C., Warrick, H. M., Spudich, J. A., and Leinwand, L. A. (2010). Functional diversity among a family of human skeletal muscle myosin motors. Proc. Natl. Acad. Sci. U S A. 107, 1053-1058. doi: 10.1073/pnas. 0913527107

Robbins, J., Gangnon, R. E., Theis, S. M., Kays, S. A., Hewitt, A. L., and Hind, J. A. (2005). The effects of lingual exercise on swallowing in older adults. J. Am. Geriatr. Soc. 53, 1483-1489. doi: 10.1111/j.1532-5415.2005.53467.x

Robbins, J., Hamilton, J. W., Lof, G. L., and Kempster, G. B. (1992). Oropharyngeal swallowing in normal adults of different ages. Gastroenterology 103, 823-829. doi: 10.1016/0016-5085(92)90013-O

Robbins, J., Levine, R., Wood, J., Roecker, E. B., and Luschei, E. (1995). Age effects on lingual pressure generation as a risk factor for dysphagia. J. Gerontol. Ser. A Biol. Sci. Med. Sci. 50, M257-M262. doi: 10.1093/gerona/50A.5.M257

Rogus-Pulia, N., Rusche, N., Hind, J. A., Zielinski, J., Gangnon, R., Safdar, N., et al. (2016). Effects of Device-Facilitated Isometric Progressive Resistance Oropharyngeal Therapy on Swallowing and Health-Related Outcomes in Older Adults with Dysphagia. J. Am. Geriatr. Soc. 64, 417-424. doi: 10.1111/jgs.13933

Rosenberg, I. H. (1997). Sarcopenia: origins and clinical relevance. J. Nutrit. 127(5 Suppl.), 990s-991s. doi: 10.1093/jn/127.5.990S

Schaser, A. J., Ciucci, M. R., and Connor, N. P. (2016). Cross-activation and detraining effects of tongue exercise in aged rats. Behav. Brain Res. 297, 285-296. doi: 10.1016/j.bbr.2015.10.030

Schaser, A. J., Wang, H., Volz, L. M., and Connor, N. P. (2011). Biochemistry of the anterior, medial, and posterior genioglossus in the aged rat. Dysphagia 26, 256-263. doi: 10.1007/s00455-010-9297-x

Shune, S. E., Karnell, L. H., Karnell, M. P., Van Daele, D. J., and Funk, G. F. (2012). Association between severity of dysphagia and survival in patients with head and neck cancer. Head Neck 34, 776-784. doi: 10.1002/hed.21819

Smithard, D., O'neill, P., Park, C., and Morris, J. (1996). Complications and outcome after acute stroke: does dysphagia matter? Stroke J. Cereb. Circulat. 27, 1200-1204. doi: 10.1161/01.STR.27.7.1200

Steele, C. M., Greenwood, C., Ens, I., Robertson, C., and Seidman-Carlson, R. (1997). Mealtime difficulties in a home for the aged: not just dysphagia. Dysphagia 12, 43-50. doi: 10.1007/PL00009517

Suttrup, I., and Warnecke, T. (2016). Dysphagia in Parkinson's disease. Dysphagia 31, 24-32. doi: 10.1007/s00455-015-9671-9

Talmadge, R. J., Roy, R. R., and Edgerton, V. R. (1993). Muscle fiber types and function. Curr. Opin. Rheumatol. 5, 695-705. doi: 10.1097/00002281199305060-00002 
The National Academies. (2011). Guide for the care and use of laboratory animals, 8th Edn. Washington, D.C: The National Academies Press.

Thexton, A., Crompton, A., and German, R. (1998). Transition from suckling to drinking at weaning: a kinematic and electromyographic study in miniature pigs. J. Exp. Zool. 280, 327-343. doi: 10.1002/(SICI)1097-010X(19980401)280: 5<327::AID-JEZ2>3.0.CO;2-Q

Van Daele, D. J., McCulloch, T. M., Palmer, P. M., and Langmore, S. E. (2005). Timing of glottic closure during swallowing: a combined electromyographic and endoscopic analysis. Ann. Otol. Rhinol. Laryngol. 114, 478-487. doi: 10. $1177 / 000348940511400610$

Weijnen, J. A. (1998). Licking behavior in the rat: measurement and situational control of licking frequency. Neurosci. Biobehav. Rev. 22, 751-760. doi: 10.1016/ S0149-7634(98)00003-7

Yoshii, M., Sakiyama, K., Abe, S., Agematsu, H., Mitarashi, S., Tamatsu, Y., et al. (2008). Changes in the myosin heavy chain $2 \mathrm{a}$ and $2 \mathrm{~b}$ isoforms of the anterior belly of the digastric muscle before and after weaning in mice. Anat. Histol. Embryol. 37, 147-152. doi: 10.1111/j.1439-0264.2007.0 0813.x

Yoshikawa, M., Fukuoka, T., Mori, T., Hiraoka, A., Higa, C., Kuroki, A., et al. (2021). Comparison of the Iowa Oral Performance Instrument and JMS tongue pressure measurement device. J. Dent. Sci. 16, 214-219. doi: 10.1016/j.jds.2020. 06.005

Conflict of Interest: The authors declare that the research was conducted in the absence of any commercial or financial relationships that could be construed as a potential conflict of interest.

Publisher's Note: All claims expressed in this article are solely those of the authors and do not necessarily represent those of their affiliated organizations, or those of the publisher, the editors and the reviewers. Any product that may be evaluated in this article, or claim that may be made by its manufacturer, is not guaranteed or endorsed by the publisher.

Copyright $\odot 2021$ Glass, Figueroa, Russell, Krekeler and Connor. This is an openaccess article distributed under the terms of the Creative Commons Attribution License (CC BY). The use, distribution or reproduction in other forums is permitted, provided the original author(s) and the copyright owner(s) are credited and that the original publication in this journal is cited, in accordance with accepted academic practice. No use, distribution or reproduction is permitted which does not comply with these terms. 